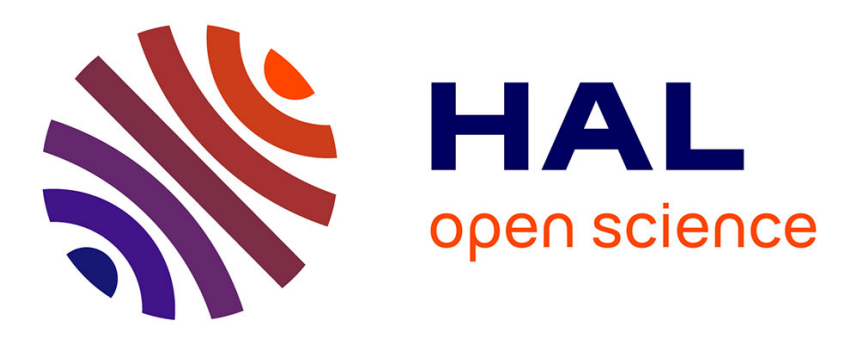

\title{
Alpha-linolenic acid: A promising nutraceutical for the prevention of stroke
}

\author{
Carine Nguemeni, Elsa Gouix, Miled Bourourou, Catherine Heurteaux, \\ Nicolas Blondeau
}

\section{- To cite this version:}

Carine Nguemeni, Elsa Gouix, Miled Bourourou, Catherine Heurteaux, Nicolas Blondeau. Alphalinolenic acid: A promising nutraceutical for the prevention of stroke. PharmaNutrition, 2013, 1 (1), pp.1-8. 10.1016/j.phanu.2012.12.002 . hal-02266098

\section{HAL Id: hal-02266098 https://hal.science/hal-02266098}

Submitted on 13 Aug 2019

HAL is a multi-disciplinary open access archive for the deposit and dissemination of scientific research documents, whether they are published or not. The documents may come from teaching and research institutions in France or abroad, or from public or private research centers.
L'archive ouverte pluridisciplinaire HAL, est destinée au dépôt et à la diffusion de documents scientifiques de niveau recherche, publiés ou non, émanant des établissements d'enseignement et de recherche français ou étrangers, des laboratoires publics ou privés. 


\section{Alpha-linolenic acid: a promising nutraceutical for the prevention of stroke}

Carine Nguemeni ${ }^{1,2}$, Elsa Gouix ${ }^{1,2}$, Miled Bourourou ${ }^{1,2}$, Catherine Heurteaux ${ }^{1,2}$ and ${ }^{*}$ Nicolas Blondeau ${ }^{1,2}$

${ }^{1}$ Institut de Pharmacologie Moléculaires et Cellulaires - UMR7275, C.N.R.S, 06560, Valbonne, France;

${ }^{2}$ Université de Nice Sophia Antipolis, 28, avenue de Valrose, Nice Cedex 2, France;

*Corresponding Author: Nicolas Blondeau

Institut de Pharmacologie Moléculaires et Cellulaires UMR7275, C.N.R.S, 06560, Valbonne, France

Telephone: +33493957740

Telefax: +33493957708

Mail: Blondeau@ipmc.cnrs.fr

Running title

$\underline{\text { ALA as a nutraceutical against stroke-induced brain lesion }}$ 


\section{Abbreviations}

ALA : alpha linolenic acid

ANSES : Agence Nationale pour la Sécurité et la Santé

BDNF : Brain Derived Neurotrophic Factor

DHA : docosahexaenoic acid

EPA : eicosapentaenoic acid - IFSSAL :

LA : linolenic acid

MCAO : middle cerebral artery occlusion

PUFA : polyunsaturated fatty acids

SNAP-25 : Synaptosomal-Associated Protein 25

VAMP-2 : Vesicle-Associated Membrane Protein 2

VGLUT1 : Vesicular Glutamate Transporter 1

VGLUT2 : Vesicular Glutamate Transporter 2 


\begin{abstract}
:
Stroke is a worldwide main cause of mortality and morbidity. Most of the preventive and neuroprotective treatments identified in preclinical studies failed in clinical trials. Although there is a consensus that nutrition is important for health, its role is underestimated in stroke. Indeed an increase consumption of salt and fatty foods may promote hypertension and obesity, which are well known risk factors of stroke. In contrast it is more difficult to identify a risk factor arising from a deficiency in an essential nutrient in the diet. Western modern diets are deficient in omega-3 polyunsaturated fatty acids, which are essential for brain health. Such deficiency may constitute by itself a risk factor for stroke. Furthermore, an imbalance in the consumption of omega- 6 and omega- 3 progressively took place in the past 40 years leading to omega-6/omega-3 ratios that are far above the WHO healthy recommendations. A consequence of this imbalanced ratio has been the fostering of elevations in and increased prevalence of inflammatory cardiovascular diseases and obesity. In this context, this review outlines a promising therapeutic opportunity by integrating a nutritional-based approach focusing on omega-3 alpha-linolenic acid as nutraceutical to prevent the devastating damage caused by stroke.
\end{abstract}

\title{
Keywords:
}

stroke - brain ischemia - neuroprotection - omega-3 polyunsaturated fatty acids - alphalinolenic acid - synaptogenesis - neurogenesis - nutraceutical - enriched diet 


\section{Introduction}

Stroke is a worldwide main cause of mortality and morbidity. It causes a significant socioeconomic cost and a marked increase in patient/family burden. More than 1000 molecules have demonstrated brain-protective properties in experimental models. More than 200 clinical trials have examined neuroprotection for ischemic stroke, arguably despite sufficiently persuasive pre-clinical justification. To date most of the preventive treatments and neuroprotective drugs identified in preclinical studies failed in clinical trials. Therefore testing alternative protective strategies to prevent stroke is of considerable importance. Although there is a consensus that nutrition is important for health, its role is underestimated in stroke prevention. It is recognized that an increase consumption of salt, fatty foods and alcoholic beverages may promote pathologies like hypertension, obesity and alcoholism, all of which are well known risk factors of stroke. In contrast it is more difficult to identify a risk factor arising from a deficiency in an essential nutrient in the diet. Western modern diets are deficient in omega-3 polyunsaturated fatty acids, which are essential for brain health. Such deficiency constitutes by itself a risk factor for cardiovascular and cerebral diseases, including coronary heart disease and stroke. Furthermore, an imbalance in the consumption of omega-6 and omega-3 PUFAs progressively took place in the past 40 years leading to omega-6/omega3 ratios that are far above the WHO healthy recommendations. A consequence of this imbalanced ratio has been the fostering of elevations in and increased prevalence of inflammatory cardiovascular and neuronal diseases and obesity. In this context, nutritional products with health benefits represent a growing interest. Functional foods enriched in omega-3 polyunsaturated acids or omega-3 used as nutraceuticals may reduce the frequency of strokes through preservation of both arterial and neuronal function. This review outlines a promising therapeutic opportunity by integrating a nutritional-based approach focusing on enriching the omega-3 alpha-linolenic acid (ALA) to prevent the devastating damage caused by stroke.

\section{Stroke statistics and healthcare}

\subsection{Stroke statistics}

Stroke is the third leading cause of mortality and disability in the world. It has been predicted that stroke will account for $6.2 \%$ of the illness total burden in the first nine years in 2000 [1]. The mortality rate at five years is approximately $50 \%$ and a stroke strikes every 40 seconds with one death every four minutes [2,3]. The total number of stroke deaths is estimated at 
508,000 per year in European Union; among the elderly population of 15 European countries, surveys showed 2,700,000 prevalent cases, and 536,000 incident cases each year [4]. In the United States, data show a total of 5,800,000 prevalent stroke cases, with 780,000 being first occurrences or recurrent strokes expected each year. Stroke causes many impairments and half of stroke survivors are left to live with physical or cognitive disabilities. This gives rise to real issues with hospital organization, care and public health, invariably exerting a high cost and a burden to society and health systems [5]. Because stroke compromises patients' lives so severely, they will require high levels of assistance and support even for common daily activities, which in turn directly impacts their quality of life - and their supporters. Therefore, those indirect costs contribute to increase the economic burden associate with stroke. The total economic costs (direct and indirect cost) for stroke were estimated at $\$ 73.7$ billion in 2010 [3]. The only current therapy against stroke is thrombolysis, which is provided only given to $\sim 5 \%$ of patients because of 1 ) its numerous side effects, the major one being a $6-7 \%$ risk of bleeding [6] and;2) the therapeutic window of 3 to 4.5 hours required to obtain a therapeutic effect of the thrombolytic [7]. Generally, the majority of patients are not treated with rtPA because they arrive after that narrow time window of treatment of opportunity, leaving clinicians without any repertoire of therapeutic opportunities. It is somewhat ironic that clot busters is considered the best 'neuroprotectant', given that it mediates its effect through the vasculature, as opposed to exerting any direct protection in neurons. In a landmark analysis published in 2006, none of the 114 treatments (from more than a thousand treatments demonstrating neuroprotection against acute stroke since the $1960 \mathrm{~s}$ at the preclinical level) achieved neuroprotection in clinical trials [8]. In addition, these drugs displayed poor adherence to the STAIR criteria (Stroke Therapy Academic Industry Roundtable), designed to increase the rigor by which neuroprotective agents were assessed at the preclinical level, in order to improve the odds of success in clinical trials [9]. This failure in translation from experimental models to clinical trials led to a major exodus of pharmaceutical companies from stroke, and also prompted a major re-evaluation of properties, which would constitute the "best-in class" therapeutics to be used against stroke, integrating several aspect of the pathology of stroke.

\subsection{Stroke physiopathology}

An ischemic stroke is the result of an occlusion within a blood vessel supplying blood to the brain. This type of stroke accounts for more than $\sim 80 \%$ of all stroke cases. The current 
understanding of its pathophysiology has dramatically evolved over the past two decades mainly due to the animal studies. It is well known that the clot may either be formed locally in a small artery or may arise from the heart or from an injury in the wall of one of the cervical arteries (carotid and vertebral arteries). Occlusion of the middle cerebral artery (MCA) is one of the most widely used experimental models. Interruption of blood flow generated a hypoperfusion in the MCA territory that leads to a rapid decline in oxygen (hypoxia) and nutriments (glucose, ATP, energetic metabolites...). The cut off the supply of oxygen and glucose prevents the brain from generating the ATP needed to support its considerable energy demands. After focal ischemia, this energy deficit is most severe in the core of the ischemic territory that displays the lowest residual flow, wherein cell death occurs rapidly. In the core periphery, termed ischemic penumbra, even if reduced collateral blood flow buffers the full effects of stroke, waves of peri-infarct depolarization lead to neurotransmitter release. In concert with impairment of glial reuptake, extracellular glutamate achieves neurotoxic concentrations. Within the entire ischemic territory, glutamate overload into the extracellular space results in an hyperactivation of its receptors (excitotoxicity) leading to accumulation of intracellular $\mathrm{Ca}^{2+}$, which in turn sets off deleterious events including activation of lytic enzymes, mitochondrial dysfunction, and oxidative stress [9-11]. Irreversible damages are caused to neuronal membrane and compromise brain tissue survival. Initiation of these pathologic pathways differs in space and time (Figure 1) and also depends of the cellular subtype (neuronal or vascular, for example) [12].

Historically, stroke research aimed to target one element of the deleterious cascade that takes place during stroke [8]. It failed to identify and validate an effective drug able to protect the neurovascular unit. Although restoration of cerebral blood flow can be successfully applied in a small 5\% subset of patients by recombinant tissue plasminogen activator, there is still no therapeutic for stroke, underlining a pressing need to investigate additional strategies. Epidemiologic studies have demonstrated most patients presenting with a stroke possessed at least one risk factor. In addition, the pre-existence of certain risk factors in the patient history may worsen stroke outcome and compromises seriously post-stroke rehabilitation. These risk factors may also compromise also the patient eligibility to thrombolysis therapy [13]. Therefore, prevention of risk factors is one of the emerging axes of stroke care.

\subsection{Stroke, risk factors and nutrition}


Severable modifiable and non-modifiable risk factors for ischemic stroke have been identified. Non-modifiable and irreversible risk factors include age, gender, ethnicity, and heredity. Among modifiable risk factors are hypertension, hypercholesterolemia, alcohol, smoking, atrial fibrillation, obesity and diabetes, which are in constant progression in all industrialized countries. The coexistence of modifiable risk factors has been estimated at up to $80 \%$ of stroke incidence [14]. It is worth noting that modifiable risk factors of stroke often coexist with improper life-style and poor nutrition, causing imbalances in essential vitamins and nutriments, such as polyunsaturated fatty acids (PUFA) from the omega-3 family. Many clinical and epidemiologic studies have shown that insufficient dietary intake of fruits and vegetables causing deficiencies in vitamins, nutrients, and essential omega-3 polyunsaturated fatty acids (PUFAs) may be risk factor for cardiovascular and cerebral diseases, including stroke [15]. It is now clear that the evolution of human diet and the modernization of food manufacturing processes account for most of the essential nutrient deficiencies in our diet. As an example, the western diets provide an excessive quantity of omega- 6 at the expense of omega-3. In the majority of the adult population of developed countries, omega-3 intake is far below the recommended Dietary Reference Intake, while consumption of omega- 6 is more than 10 times higher than for omega-3. Consequently, the reported omega-6/omega-3 ratio is often above 10-15, compared to a ratio of approximately 5 recommended by most of the international Health organizations [16]. This elevated ratio represents strong evidence of an imbalance in the intake of these two families of essential fatty acids. Several epidemiologic studies demonstrated beneficial effects of diets enriched in omega-3 on various cerebral diseases," also suggesting that they may reduce the risk of stroke. Meanwhile, the few preclinical studies that addressed their potential protective role against stroke-induced damage suggest that the nutraceutical potential of omega-3 could be a field of study of potentially major relevance. Altogether it emphasizes the need to focus more closely on the members of the omega-3 family and their nutraceutical potential in stroke.

\section{Why the polyunsaturation matter in omega-6 and omega-3}

\subsection{Omega-6/ omega-3 structure}

Omega-3 and omega-6 PUFA families are structurally similar, with both containing a carbon long chain linked to hydrogen atoms punctuated by double bonds (unsaturation) and terminated by an acidic group. The conventional nomenclature differentiates the chemical structure of omega- 3 and omega- 6 by the location of the first double bond that is marked from 
the methyl-terminal extremity. All fatty acids contain their first double bound between the $6^{\text {th }}$ and $7^{\text {th }}$ carbon atoms in the omega- 6 family, and between the $3^{\text {rd }}$ and $4^{\text {th }}$ carbon atom in the omega-3 fatty acids. Mammals cannot synthesize alpha-linolenic (ALA, C18:3 133 ) and

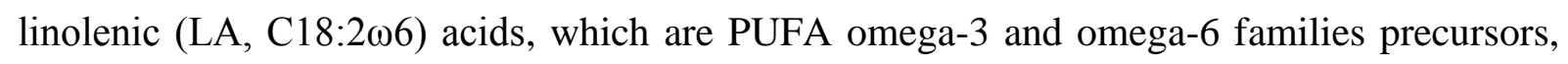
respectively. By analogy with essential amino acids, ALA and LA are essential fatty acids and have to be supplied by the diet [17]. LA metabolism leads to the production of arachidonic acid (AA, C20:4 n-6) and docosapentaenoic acid (C22:6 n-6). ALA is successively transformed to eicosapentaenoic acid (EPA, C 20:5 n-3) which converts to docohexaenoic acid (DHA, C22:6 n-3). In mammals, the same enzymes (desaturases and elongases) participate in the synthesis of omega-3 and omega-6 long chain PUFA (figure 2). This results in a competition between both families to access the enzymatic pathway. The bioconversion rate of ALA to EPA and DHA is extremely low, accounting for why the omega-3 long chain are also considered as practically essential since they are almost all supplied by the diet [18]. Thus, any excess in LA intake may compromise EPA and DHA bioconversion from ALA. Moreover, enzymes involved in the metabolism of LA and ALA (elongases and desaturases) have a high preference for LA [19]. Their activity is inhibited by risk factors for stroke such as sugar, alcohol, saturated fat, aging and diabetes that are all well described. Therefore, any change in our diet, especially in the diary intake of LA and ALA impacts the ratio omega6/omega-3, raising concerns of omega-3 levels being too low to support protection or resistance against disease development. It is acknowledged that omega- 6 and omega- 3 fatty acids should be consumed in a balanced manner, from a variety of sources (vegetal and animal fats). Recommendations made based on epidemiologic studies insist on the necessity restoring the balance omega-6/omega- 3 to a ratio of 5:1. Hence, the need for a diet enriched in the different categories of omega-3 seems necessary to assure adequate intake of essential fatty acids to restore that balance.

The large availability and the low cost of food sources containing trans fatty acids that are now recognized as detrimental to health facilitate their consumption in excess. In the same vein, human major food sources of PUFAs are omega-6-rich and omega-3-poor. Moreover, the nature of the omega-3 source shapes the content in omega-3 PUFAs. Animal sources that are rich in omega-3 are mostly fish. They provide EPA and DHA and display an omega6/omega-3 ratio extremely favorable to omega-3. Although animal sources could be used to counteract an unbalanced diet, they are often deprived of ALA. On the contrary vegetable sources of omega-3 may be rich in ALA but not in EPA and DHA and only linseed, rapeseed 
and walnut oils furnish an omega-6:omega-3 ratio corresponding to the WHO recommendations (inferior to 5, Table 1). ALA is found in diets enriched in vegetable such as red kidney beans and lima beans. Vegetable oil is one of the most important sources of PUFA (Table 1). However, several of the most commonly used oils have very low levels of PUFA (e.g. palm oil) and often very low levels of ALA (e.g. sunflower oil, sesame oil, and palm oil) [20].

\subsection{The omega-3 family: DHA and EPA versus ALA supplementation}

Although the term nutraceutical is commonly used in marketing, it has no regulatory definition. According to DeFelice, who first proposed the term coined from "nutrition" and "pharmaceutical", nutraceutical can be defined as a food (or food extract) that provides medical or health benefits, including the prevention and/or treatment of a disease. As compared to functional food or dietary supplements that support the body with the required amount of vitamins, fats, proteins, carbohydrates, etc, needed for its proper functioning, nutraceutical, which may be used in functional food or supplements should also have a proven efficiency preventing and/or treating disease [21]. The current approach to establish the efficacy of nutraceutical or nutritional interventions suffers from several limitations. We believe for clarity sake, that the used of nutraceutical may be restricted to compound isolated or purified from foods, and that could be used in medicinal forms. In this context, the interest for omega-3 as a nutraceutical has been focused mainly on long chain PUFA, EPA and DHA more than their precursor, ALA. Several reasons support this trend. A major reason is that ALA bioconversion to DHA seems very limited, with many studies reporting that conversion efficiency from ALA to its immediate metabolite EPA is $0.4 \%$ and the overall efficiency of conversion to DHA is less than $0,1 \%$ [22,23]. Diet supplementation with high levels of ALA leads to small increases in EPA (5\%) as compared to supplementation with preformed EPA that is approximately 15-fold more efficient [18]. Barcelo-Coblijn and Murphy (2009) published a detailed review which examined publications studying conversion of ALA to EPA and DHA. Their conclusion was that exclusive consumption of ALA could not fulfill DHA requirements [24]. It is also often suggested that EPA and DHA combination may be more efficient in reducing the cardiovascular risk effect than ALA [25,26]. But, as shown in numerous studies, ALA exerts identical effects as DHA in different physiological processes, although longer periods and higher amounts of consumption of ALA are probably required [24]. Unfortunately, while most of the studies reported biophysical and functional properties 
of DHA and EPA, intrinsic properties of ALA have not been highlighted and its potential as nutraceutical, a field of study of potential major relevance has been inadequately addressed.

\subsection{Beneficial effects of ALA: the origin of the concept}

The pioneering studies of Tinoco and colleagues demonstrated important functions of ALA in animals. ALA deficiency in rat alimentation over several generations causes vision and brain function impairments [27]. Since that point, some in vitro and in vivo studies examining the effect of ALA on neurons directly demonstrated its potential against glutamate-mediated excitotoxicity, a neurodestructive phenomenon shared by many acute and chronic neurodegenerative diseases including epileptic seizures, acute spinal cord injury (SCI) and stroke [15]. In an experimental model of SCI in rats, ALA treatment 30 min before trauma reduced lesion size by $45 \%$ and improved neuronal survival and locomotor activity measured one week after injury [28]. The beneficial effects of ALA on function, neuronal/glia survival and biochemical marker in various SCI models were reviewed in animals subjected to SCI $[29,30]$. In a model of epilepsy induced by kainate administration, a powerful activator of glutamate receptor system, ALA prevented neurotoxicity and seizure-like activity [31,32]. In vitro, ALA reduced hippocampal neuronal death induced by excitotoxic concentration of glutamate [33]. Finally, chronic intake of the "SR-3", a fatty acid compound with LA/ALA ratio of 4, promoted neuroprotection against pentylenetetrazol-induced seizures in rat [34].

Despite the fact that a direct link between ALA deficiency and cardiovascular disease has not been clearly demonstrated to date, epidemiological and correlations studies outlined its potential in cardiovascular diseases. Mediterranean and Japanese populations that consume foods rich in ALA are more resistant to cardiovascular disease [35].

Djoussé et al. have investigated whether ALA consumption was associated with a lower risk of carotid atherosclerosis. Dietary ALA was inversely related to the thickness of carotid arteries, whereas fish-EPA and DHA consumption was not, suggesting that ALA could prevent the progression of atherosclerosis [36]. In addition to the protective effect of ALA against atherosclerotic plaque in coronary artery, dietary ALA prevented hypertension [36]. With regard to stroke, ALA plasma level increases seems to be correlated with a $37 \%$ decrease of the stroke risk [37]. These results indicate that ALA may play a role in the prevention or therapy of cardiovascular and neurovascular diseases.

\section{ALA and stroke}


We have stressed the fact that the lack of omega-3 fatty acid could result in serious consequences for heart and brain health. As for cardiovascular diseases, many strokes caused by unhealthy diet and lifestyle could potentially be avoided. In this context, and in view of the recurrent failures in the development of drugs to prevent stroke, nutraceuticals or functional food enriched with omega-3 like ALA may represent a promising opportunity in prevention and perhaps also in treatment of neurovascular insults.

\subsection{ALA and the neurovascular unit}

Our interest in omega-3 originated from our discovery of their ability to activate the potassium channel TREK-1, regarded as a promising molecular target for new neuroprotective strategies [38]. Electrophysiology experiments revealed that ALA and its long chain derivatives EPA and DHA activate this channel, leading to cell hyperpolarization that may counteract lethal neuronal depolarizations associated with glutamate excitotoxicity, a key mechanism of stroke-induced neurodegeneration [32]. Although the protection may involve calcium, sodium and potassium conductance regulation, TREK-1 activation is compulsory for ALA-induced protection against stroke. In contrast, omega-6 PUFA like LA and AA seem less efficient in activating TREK-1 potassium channels and saturated fatty acids like palmitate, stearate and arachidate have no effects. In animal models reproducing the cerebral consequence of a myocardial arrest and epileptic seizure - a regular consequence in stroke omega-3 acute injection reduced neuronal loss [31,32]. ALA cerebral protection is probably mediated by a direct effect on neurons, although ALA may exert its beneficial effect acting on the vascular compartment. Stroke injury also affects vascular functions altering the tonus of the injured artery. The loss of vasodilation capacity compromises the perfusion of the cerebral tissue. In vitro, ALA application onto basilar artery promotes its vasodilatation. This effect is specific to resistance arteries and does not extend to the carotid artery, explaining the absence of systemic effect on arterial pressure $[39,40]$. Cerebral blood flow measurement by laser Doppler revealed that injection of neuroprotective dose of ALA increased rates in rodents, as compared to the saturated fatty acids palmitate. ALA is not only beneficial to neurons but is also of great interest also for blood vessels. This implies that ALA could be beneficial for the whole neurovascular compartment, compared to a large number of typical stroke neuroprotective chemical molecules which only target neurons, and have failed in clinical trials. ALA might represent a successful neuroprotectant where many had tried and failed.

\subsection{ALA in an experimental model of focal ischemic stroke}


In hypertensive rats highly predisposed to stroke, Shimokawa and colleagues highlighted that a diet enriched in perilla oil (60\% ALA) increases rat lifetime, compared to a diet enriched in sunflower oil (rich in linolenic acid) [41]. In humans, a clinical study conducted in 192 patients revealed a strong correlation between ALA plasma levels in patients and a reduction in stroke susceptibility [37]. In humans, the occlusion of the sylvian artery represents about $70 \%$ of stroke events. In a mouse model of ischemic stroke by the transient occlusion of the middle cerebral artery (MCAo), we investigated whether the intravenous injection of ALA could reduce the infarct volume. Our results show that ALA injection is effective when injected up to 6 hours after stroke onset, a time-frame probably compatible with a clinical setting. Those results are similar to those obtained with riluzole, a well described neuroprotectant, delivered in amyotrophic lateral sclerosis, suggesting that a nutraceutical like ALA could perform as a chemical. Interestingly, a single ALA post-treatment did not improve long term survival, but several staggered post-treatment ALA injections improved the survival rates three fold measured one month after stroke onset [42]. This chronic ALA treatment when used as pre-treatment also reduces the post-ischemic infarct 24 hours after MCAo [33].

\subsection{ALA supplementation, synaptogenesis and neurogenesis}

The crucial evidence that ALA needs to be chronically injected to promote long term benefit suggests that ALA-induced protection against stroke is not exclusively built on its capacity to reduce glutamate-mediated excitotoxicity, a transient phenomenon that takes place during the first few hours post-ischemia. Since we have showed that omega-3 might stimulate endogenous protective pathways $[31,33,43]$, it underlines the necesitty to dissect the subchronic ALA effects per se. We discovered that ALA treatment promotes neuronal plasticity, which may contribute to brain repair after stroke. Indeed this subchronic ALA treatment per se increases neurogenesis in the dentate gyrus. The neurogenesis accompanies an increase in synaptogenesis in the Dentate Gyrus, but also in the cortex as shown by an increase in the expression of proteins involved in synaptic function. ALA treatment induces VAMP-2 (Vesicle-Associated Membrane Protein 2, also called synaptobrevin) and SNAP-25 (Synaptosomal-Associated Protein 25) expression, which are involved in vesicle budding in the synaptic active area. Synaptophysin-1, a protein of the vesicular membrane participating to presynaptic fusion pore involved in neurotransmitter release, is also upregulated as well as the glutamate transporter expression (VGLUT1 and VGLUT2). These effects are strongly correlated with an increase of BDNF (Brain Derived Neurotrophic Factor) observed both in 
the brain of treated mice in vivo and in neural stem cells or in hippocampal cultures in vitro. Thus, the ALA protective effects mediated by a direct action on the neurovascular unit may also be supported by the stimulation of endogenous mechanisms involved in regeneration and recovery.

\section{$\underline{\text { 3.3. Dietary ALA against stroke }}$}

Since ALA supplementation by i.v. route is not relevant for prevention, we studied the possibility of providing ALA by a nutritional approach, by using an ALA-enriched vegetable oil such as rapeseed oil (9\% ALA) to attain cerebral protection similar to that attained by ALA chronic injections.

A 6-week diet enriched in ALA by a factor of three compared to regular chows and an omega6/omega-3 ratio blow 5- reduced cerebral infarct volume. The rapeseed oil-diet decreased the mortality rate three-fold and increased the spontaneous reperfusion probability measured $24 \mathrm{~h}$ post-MCAo [44]. This diet also decreased lipid peroxidation and oxidative stress associated with the brain ischemia. Beneficial effects were similar to those of parenteral supplementation and may be more efficient than those obtained by a supplementation with fish oil enriched in EPA/DHA, a paradigm still debated [44-47]. The rapeseed oil diet does not contain EPA and DHA; as mentioned previously, ALA is not sufficiently converted to DHA, so the ALAassociated protection probably does not involve a bioconversion of ALA in EPA/DHA.

\section{Conclusion}

Classic neuroprotectors failed in stroke clinical trials. Researchers now focus on molecules acting on the whole neurovascular compartment and are searching for neuroprotectantpromoting protective mechanisms capable of acting at different intervals to counteract the different pathologic pathways. Then, it is tempting to speculate that ALA may adhere to the criteria of being a pleiotropic molecule (figure 3), able to block the deleterious neurotoxic signaling cascades in neurons, stimulate vascular function and also to stimulate defense and recovery mechanisms. The nutritional importance of ALA and its pleiotropic benefits should lead the scientific and medical community to focus interest in this particular omega 3 in other neurologic pathologies.

\section{$\underline{\text { 5. Acknowledgement }}$}


The GLN, ONIDOL, the Fondation de la Recherche Médicale and the CNRS supported this work. The authors thank Dr Bernadette Delplanque for many helpful discussions and all our past and present team members, especially Julia Magnin and collaborators who have contributed to the data discussed in the review.

\section{Conflict Of Interest}

None

\section{Legend of the figures}

Table 1: linoleic and alpha- linolenic acids composition of plant oil (g/100g), modified from Michaelsen et al. 2011 [20].

The type of omega-3 PUFA is a function of their origin (animal versus vegetable). Animal sources of omega-3 provide EPA/DHA and display an omega-3/omega-6 ratio in accordance to nutritional recommendations, but are often deprived of ALA. In contrast, vegetable sources of omega-3 are rich in ALA but not EPA/DHA. Only linseed, rapeseed and walnut oils offer a omega-3/omega-6 ratio lower than the recommended limit of 5. LA, linoleic acid; ALA, Alpha-linolenic acid; PUFA, polyunsaturated fatty acid.

Figure 1: Initiation of stroke induced-pathologic signaling pathways differs in space and time, modified from Dirnagl 1999 [12]

The $\mathrm{x}$-axis reflects the evolution of the cascade over time, while the $\mathrm{y}$-axis illustrates the impact of each event of the cascade on final outcome. Rapidly after the onset of the focal perfusion deficit, excitotoxic mechanisms trigger primarily neuron but also glia necrosis. In addition, excitotoxicity initiates a number of events leading to the demise of the brain tissue. This includes peri-infarct depolarizations and more delayed neurotoxicity mechanisms such as inflammation and programmed cell death. A brain region experiencing low rates of perfusion in which cells have lost their membrane potential terminally (core) is surrounded by an area in which intermediate perfusion prevails (penumbra) and cells depolarize intermittently (periinfarct depolarization). Note that from the onset of the focal perfusion deficit, the core and penumbra have different neurotoxic mechanistic dynamics in space and timing.

Figure 2: Biosynthesis of the omega-6 and omega-3 PUFA. 
The omega- 6 and omega- 3 families possess structural similarities. In mammals, the synthesis of the long chain derivatives from their distinct precursors LA and ALA involves the same enzymes (desaturases and elongase). This creates a competition between both families to develop the metabolic derivatives. LA is desaturated to form gamma-linolenic acid (GLA, 18:3 omega-6) that in turn is elongated to form GLA (20:3 omega-6), the precursor of one series of prostaglandins (PGs). GLA is also converted to form AA (arachidonic acid, 20:4 omega-6), the precursor of 2 series of PGs, thromboxanes (TXs) and 4 series leukotrienes (LTs). Similarly, ALA is converted to eicosapentaenoic acid (EPA, 20:5 omega-3), the precursor of 3 series of PGs, TXs, and 5 series LTs. EPA is also converted to form docosahexaenoic acid (DHA, 22:6 omega-3). Thus, excessive intake of LA can compromise eicosanoid acid (EPA) and docohexaenoic (DHA) production from ALA, the major long chains PUFA.

\section{Figure 3: ALA potential on different level of stroke-induced damages}

The schematic summarizes the different properties of ALA that could counteract strokeinduced damage. 


\section{REFERENCES}

1 Menken M, Munsat TL, Toole JF. The global burden of disease study: Implications for neurology. Arch Neurol 2000;57:418-420.

2 Lloyd-Jones D, Adams RJ, Brown TM, Carnethon M, Dai S, De Simone G, Ferguson TB, Ford E, Furie K, Gillespie C, Go A, Greenlund K, Haase N, Hailpern S, Ho PM, Howard V, Kissela B, Kittner S, Lackland D, Lisabeth L, Marelli A, McDermott MM, Meigs J, Mozaffarian D, Mussolino M, Nichol G, Roger VL, Rosamond W, Sacco R, Sorlie P, Thom T, Wasserthiel-Smoller S, Wong ND, Wylie-Rosett J. Heart disease and stroke statistics--2010 update: A report from the american heart association. Circulation 2010;121:e46-e215.

3 Rosamond W, Flegal K, Furie K, Go A, Greenlund K, Haase N, Hailpern SM, Ho M, Howard V, Kissela B, Kittner S, Lloyd-Jones D, McDermott M, Meigs J, Moy C, Nichol G, O'Donnell C, Roger V, Sorlie P, Steinberger J, Thom T, Wilson M, Hong Y. Heart disease and stroke statistics--2008 update: A report from the american heart association statistics committee and stroke statistics subcommittee. Circulation 2008;117:e25-146.

4 Di Carlo A, Launer LJ, Breteler MM, Fratiglioni L, Lobo A, Martinez-Lage J, Schmidt R, Hofman A. Frequency of stroke in europe: A collaborative study of population-based cohorts. Ilsa working group and the neurologic diseases in the elderly research group. Italian longitudinal study on aging. Neurology 2000;54:S28-33.

5 Spieler JF, Lanoe JL, Amarenco P. Costs of stroke care according to handicap levels and stroke subtypes. Cerebrovasc Dis 2004;17:134-142.

6 Kaur J, Zhao Z, Klein GM, Lo EH, Buchan AM. The neurotoxicity of tissue plasminogen activator? J Cereb Blood Flow Metab 2004;24:945-963.

7 Hacke W, Kaste M, Bluhmki E, Brozman M, Davalos A, Guidetti D, Larrue V, Lees KR, Medeghri Z, Machnig T, Schneider D, von Kummer R, Wahlgren N, Toni D. Thrombolysis with alteplase 3 to 4.5 hours after acute ischemic stroke. N Engl J Med 2008;359:1317-1329.

8 O'Collins VE, Macleod MR, Donnan GA, Horky LL, van der Worp BH, Howells DW. 1,026 experimental treatments in acute stroke. Ann Neurol 2006;59:467-477.

9 Moskowitz MA, Lo EH, Iadecola C. The science of stroke: Mechanisms in search of treatments. Neuron 2010;67:181-198.

10 Endres M, Dirnagl U. Ischemia and stroke. Adv Exp Med Biol 2002;513:455-473.

11 Moustafa RR, Baron JC. Pathophysiology of ischaemic stroke: Insights from imaging, and implications for therapy and drug discovery. Br J Pharmacol 2008;153 Suppl 1:S44-54.

12 Dirnagl U, Iadecola C, Moskowitz MA. Pathobiology of ischaemic stroke: An integrated view. Trends Neurosci 1999;22:391-397.

13 Naess H, Waje-Andreassen U, Nyland H. Risk factor burden predicts long-term mortality in young patients with arterial cerebral infarction. Acta neurologica Scandinavica 2012

14 Allen CL, Bayraktutan U. Risk factors for ischaemic stroke. Int J Stroke 2008;3:105-116. 
15 Blondeau N, Tauskela JS. A new future in brain preconditioning based on nutraceuticals: A focus on a-linolenic omega-3 fatty acid for stroke protection. In: Gidday JM, Perez-Pinzon MA, Zhang JH, eds. Innate tolerance in the cns: Springer 2012.

16 World Health Organization. Who and fao joint consultation: Fats and oils in human nutrition. Nutr Rev 1995;53:202-205.

17 Holman RT. Nutritional and metabolic interrelationships between fatty acids. Fed Proc 1964;23:1062-1067.

18 Brenna JT, Salem N, Jr., Sinclair AJ, Cunnane SC. Alpha-linolenic acid supplementation and conversion to n-3 long-chain polyunsaturated fatty acids in humans. Prostaglandins Leukot Essent Fatty Acids 2009;80:85-91.

19 Emken EA, Adlof RO, Gulley RM. Dietary linoleic acid influences desaturation and acylation of deuterium-labeled linoleic and linolenic acids in young adult males. Biochim Biophys Acta 1994;1213:277-288.

20 Michaelsen KF, Dewey KG, Perez-Exposito AB, Nurhasan M, Lauritzen L, Roos $\mathrm{N}$. Food sources and intake of n-6 and n-3 fatty acids in low-income countries with emphasis on infants, young children (6-24 months), and pregnant and lactating women. Matern Child Nutr 2011;7 Suppl 2:124-140.

21 Kalra EK. Nutraceutical--definition and introduction. AAPS PharmSci 2003;5:E25.

22 Hussein N, Ah-Sing E, Wilkinson P, Leach C, Griffin BA, Millward DJ. Longchain conversion of [13c]linoleic acid and alpha-linolenic acid in response to marked changes in their dietary intake in men. J Lipid Res 2005;46:269-280.

23 Pawlosky RJ, Hibbeln JR, Novotny JA, Salem N, Jr. Physiological compartmental analysis of alpha-linolenic acid metabolism in adult humans. J Lipid Res 2001;42:12571265.

24 Barcelo-Coblijn G, Murphy EJ. Alpha-linolenic acid and its conversion to longer chain n-3 fatty acids: Benefits for human health and a role in maintaining tissue n-3 fatty acid levels. Prog Lipid Res 2009;48:355-374.

25 Burdge GC, Calder PC. Dietary alpha-linolenic acid and health-related outcomes: A metabolic perspective. Nutr Res Rev 2006;19:26-52.

26 Wang C, Harris WS, Chung M, Lichtenstein AH, Balk EM, Kupelnick B, Jordan HS, Lau J. N-3 fatty acids from fish or fish-oil supplements, but not alpha-linolenic acid, benefit cardiovascular disease outcomes in primary- and secondary-prevention studies: A systematic review. Am J Clin Nutr 2006;84:5-17.

27 Tinoco J. Dietary requirements and functions of alpha-linolenic acid in animals. Prog Lipid Res 1982;21:1-45.

28 King VR, Huang WL, Dyall SC, Curran OE, Priestley JV, Michael-Titus AT. Omega-3 fatty acids improve recovery, whereas omega- 6 fatty acids worsen outcome, after spinal cord injury in the adult rat. J Neurosci 2006;26:4672-4680.

29 Dyall SC, Michael-Titus AT. Neurological benefits of omega-3 fatty acids. Neuromolecular Med 2008;10:219-235.

30 Michael-Titus AT. Omega-3 fatty acids and neurological injury. Prostaglandins Leukot Essent Fatty Acids 2007;77:295-300.

31 Blondeau N, Widmann C, Lazdunski M, Heurteaux C. Polyunsaturated fatty acids induce ischemic and epileptic tolerance. Neuroscience 2002;109:231-241. 
32 Lauritzen I, Blondeau N, Heurteaux C, Widmann C, Romey G, Lazdunski M. Polyunsaturated fatty acids are potent neuroprotectors. EMBO J 2000;19:1784-1793.

33 Blondeau N, Nguemeni C, Debruyne DN, Piens M, Wu X, Pan H, Hu X, Gandin C, Lipsky RH, Plumier JC, Marini AM, Heurteaux C. Subchronic alpha-linolenic acid treatment enhances brain plasticity and exerts an antidepressant effect: A versatile potential therapy for stroke. Neuropsychopharmacology 2009;34:2548-2559.

34 Rabinovitz S, Mostofsky DI, Yehuda S. Anticonvulsant efficiency, behavioral performance and cortisol levels: A comparison of carbamazepine (cbz) and a fatty acid compound (sr-3). Psychoneuroendocrinology 2004;29:113-124.

35 Simopoulos AP. The importance of the omega-6/omega-3 fatty acid ratio in cardiovascular disease and other chronic diseases. Exp Biol Med (Maywood) 2008;233:674-688.

36 Djousse L, Hunt SC, Arnett DK, Province MA, Eckfeldt JH, Ellison RC. Dietary linolenic acid is inversely associated with plasma triacylglycerol: The national heart, lung, and blood institute family heart study. Am J Clin Nutr 2003;78:1098-1102.

37 Simon JA, Fong J, Bernert JT, Jr., Browner WS. Serum fatty acids and the risk of stroke. Stroke 1995;26:778-782.

38 Heurteaux C, Guy N, Laigle C, Blondeau N, Duprat F, Mazzuca M, LangLazdunski L, Widmann C, Zanzouri M, Romey G, Lazdunski M. Trek-1, a k+ channel involved in neuroprotection and general anesthesia. EMBO J 2004;23:2684-2695.

39 Blondeau N, Petrault O, Manta S, Giordanengo V, Gounon P, Bordet R, Lazdunski M, Heurteaux C. Polyunsaturated fatty acids are cerebral vasodilators via the trek-1 potassium channel. Circ Res 2007;101:176-184.

40 Garry A, Fromy B, Blondeau N, Henrion D, Brau F, Gounon P, Guy N, Heurteaux C, Lazdunski M, Saumet JL. Altered acetylcholine, bradykinin and cutaneous pressure-induced vasodilation in mice lacking the trek1 potassium channel: The endothelial link. EMBO Rep 2007;8:354-359.

41 Shimokawa T, Moriuchi A, Hori T, Saito M, Naito Y, Kabasawa H, Nagae Y, Matsubara M, Okuyama H. Effect of dietary alpha-linolenate/linoleate balance on mean survival time, incidence of stroke and blood pressure of spontaneously hypertensive rats. Life Sci 1988;43:2067-2075.

42 Heurteaux C, Laigle C, Blondeau N, Jarretou G, Lazdunski M. Alpha-linolenic acid and riluzole treatment confer cerebral protection and improve survival after focal brain ischemia. Neuroscience 2006;137:241-251.

43 Blondeau N, Widmann C, Lazdunski M, Heurteaux C. Activation of the nuclear factor-kappab is a key event in brain tolerance. J Neurosci 2001;21:4668-4677.

44 Nguemeni C, Delplanque B, Rovere C, Simon-Rousseau N, Gandin C, Agnani G, Nahon JL, Heurteaux C, Blondeau N. Dietary supplementation of alpha-linolenic acid in an enriched rapeseed oil diet protects from stroke. Pharmacol Res 2010;61:226-233. 


\begin{tabular}{|c|c|c|c|c|c|}
\hline Vegetable oil & $\begin{array}{c}\text { Total } \\
\text { fat }\end{array}$ & $\begin{array}{c}\text { Total } \\
\text { PUFA }\end{array}$ & LA & ALA & $\begin{array}{c}\text { Ratio } \\
\text { LA/ALA }\end{array}$ \\
\hline Sunflower oil & 100.0 & 59.8 & 59.4 & 0.3 & $205 / 1$ \\
\hline Sesame oil & 100.0 & 41.2 & 40.7 & 0.5 & $85 / 1$ \\
\hline Palm oil & 100.0 & 9.3 & 9.0 & 0.2 & $48 / 1$ \\
\hline Olive oil & 100.0 & 9.5 & 8.8 & 0.7 & $13 / 1$ \\
\hline Soybean oil & 100.0 & 62.3 & 55.1 & 7.2 & $8 / 1$ \\
\hline Canola oil & 100.0 & 28.5 & 18.4 & 10.0 & $1.8 / 1$ \\
\hline
\end{tabular}

Table 1 

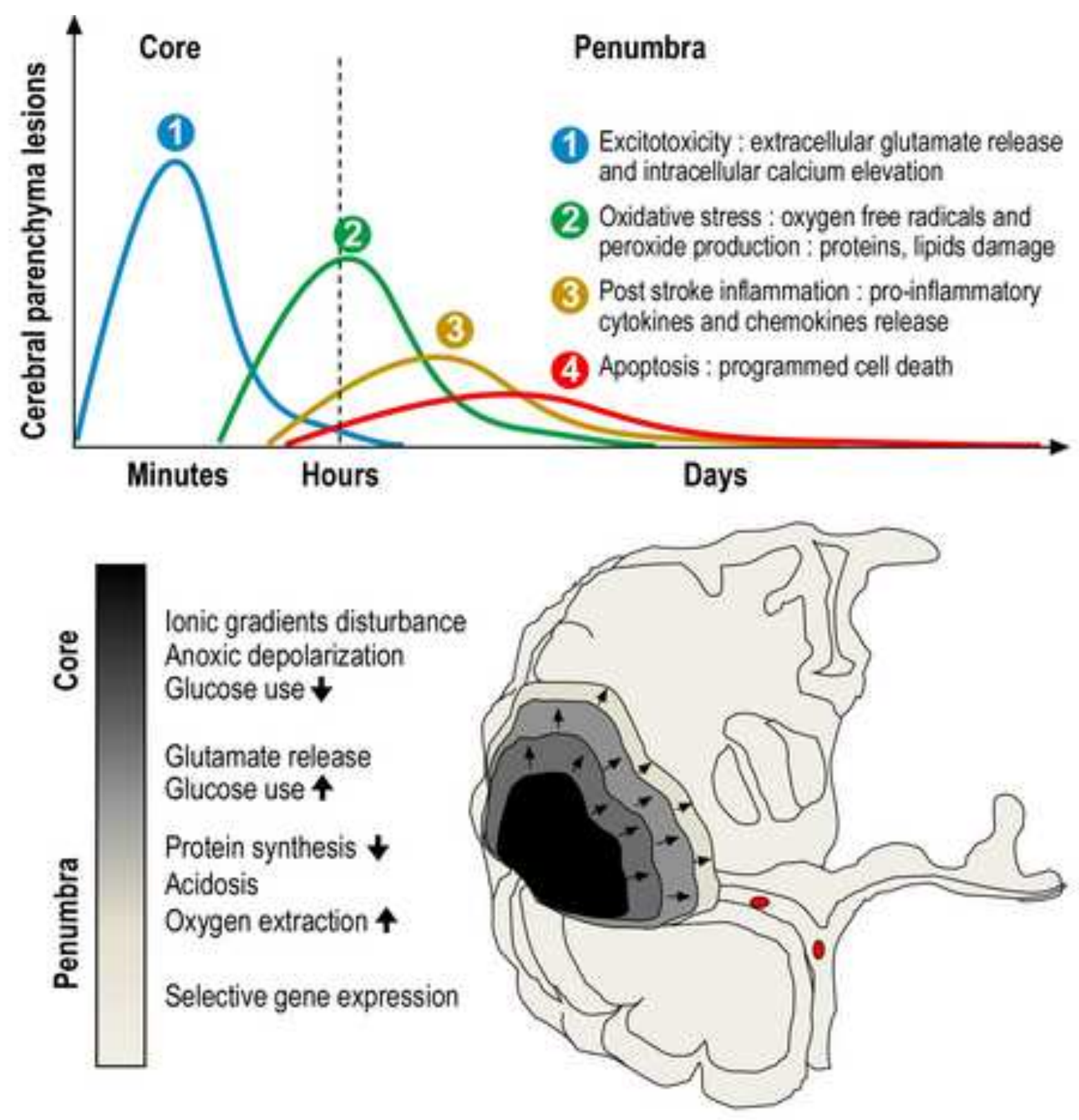

Figure 1: Initiation of stroke induced - pathologic pathways differ in space and time. 
Vegetable oils-soybean, corn oil, sunflower, safflower, and linseed oil

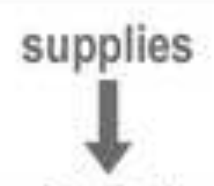

Omega-6 fatty acids

Linolenic acid (LA)

C18:20-6

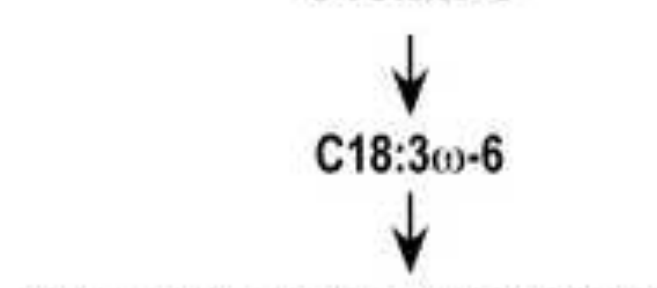

Gamma-linolenic acid (GLA) C20:3 $\omega-6$

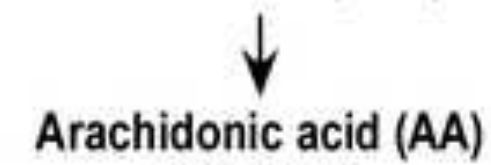

C20:3 $\omega=6$<smiles>CC(C)(C)C</smiles>

C22:4 $\omega-6$

$\downarrow$

C22:5 $\omega-6$

\section{$\Delta-6$-Desaturase}

Elongation

$\triangle$-5-Desaturase

Elongation

$\triangle$-4-Desaturase
Walnuts, rapeseed, perilla flaxseed oils and other vegetables

supplies

Omega-3 fatty acids

Alpha-linolenic acid (ALA)

C18:3 $1-3$

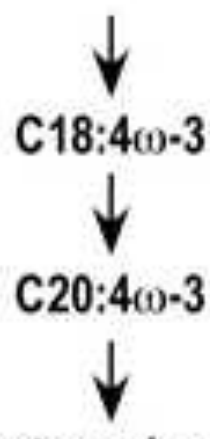

Eicosapentaenoic acid (EPA)

C20:5 $\omega-3$

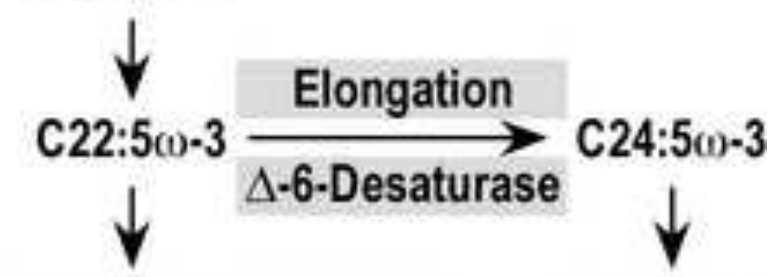

Docosahexaenoic acid (DHA)

C24:6 $\omega-3$

C22:6 $\omega-3$ 

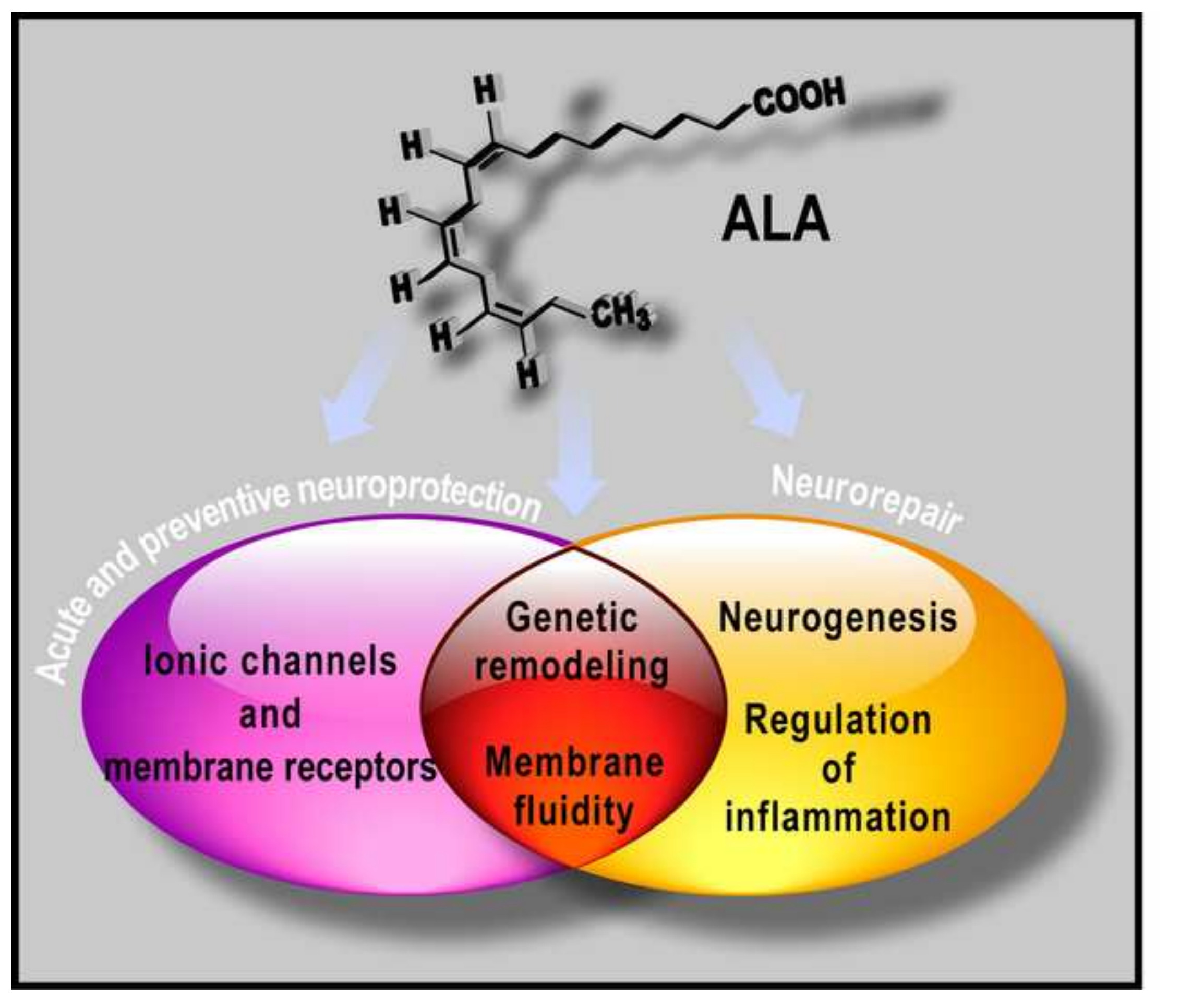

to

